\title{
Diabetes awareness in the general population of northern Greece
}

\section{Somali M. ${ }^{1}$, Daramilas Ch. ${ }^{2}$, Mastorakos G. ${ }^{3}$, Mouslech Z. ${ }^{1}$}

${ }^{1}$ National Organization for Health Care (EOPYY), Thessaloniki, Greece

${ }^{2}$ Faculty of Sciences, Department of Biology, Aristotelion University, Thessaloniki, Greece ${ }^{3}$ Department of Endocrinology, Metabolism and Diabetes, Aretaeio Hospital, School of Medicine, National and Kapodistrian University Athens, Greece

Introduction: The current study was conducted internist, $5 \%$ a general phycisian and $18 \%$ to assess the level of awareness about diabetes (DKn/NA). As causal factors $63 \%$ reported in a random population.

Methods: A questionnaire was conducted by communication over the telephone in Thessaloniki, Macedonia, Greece in a random population of 600 persons. Presence of DM1 or DM2 in the person questioned or a family member, knowledge of precipitating factors; duration, curability, choice of phycisian and compliance with medication were assessed.

Results: In the total population, $14 \%$ had diabetes. $41 \%$ reported their first choice of phycisian would be an endocrinologist, $36 \%$ an

hereditary predispotition as the primary factor and of secondary importance poor eating habits, obesity, lack of physical activity and environmental pollution. For $77 \%$ of the total population diabetes is an incurable chronic disease, for $14 \%$ an easily curable disease and $9 \% \mathrm{DKn} / \mathrm{NA}$. Among those with diabetes, $64 \%$ reported total compliance with doctors recommendations, $5 \%$ high, $15 \%$ mostly, $8 \%$ little and $5 \%$ no compliance. $28 \%$ of the population free of diabetes reported a family member with diabetes, $94 \%$ of these reported no concealment of the disease in their social behavior.
Do you have diabetes mellitus?

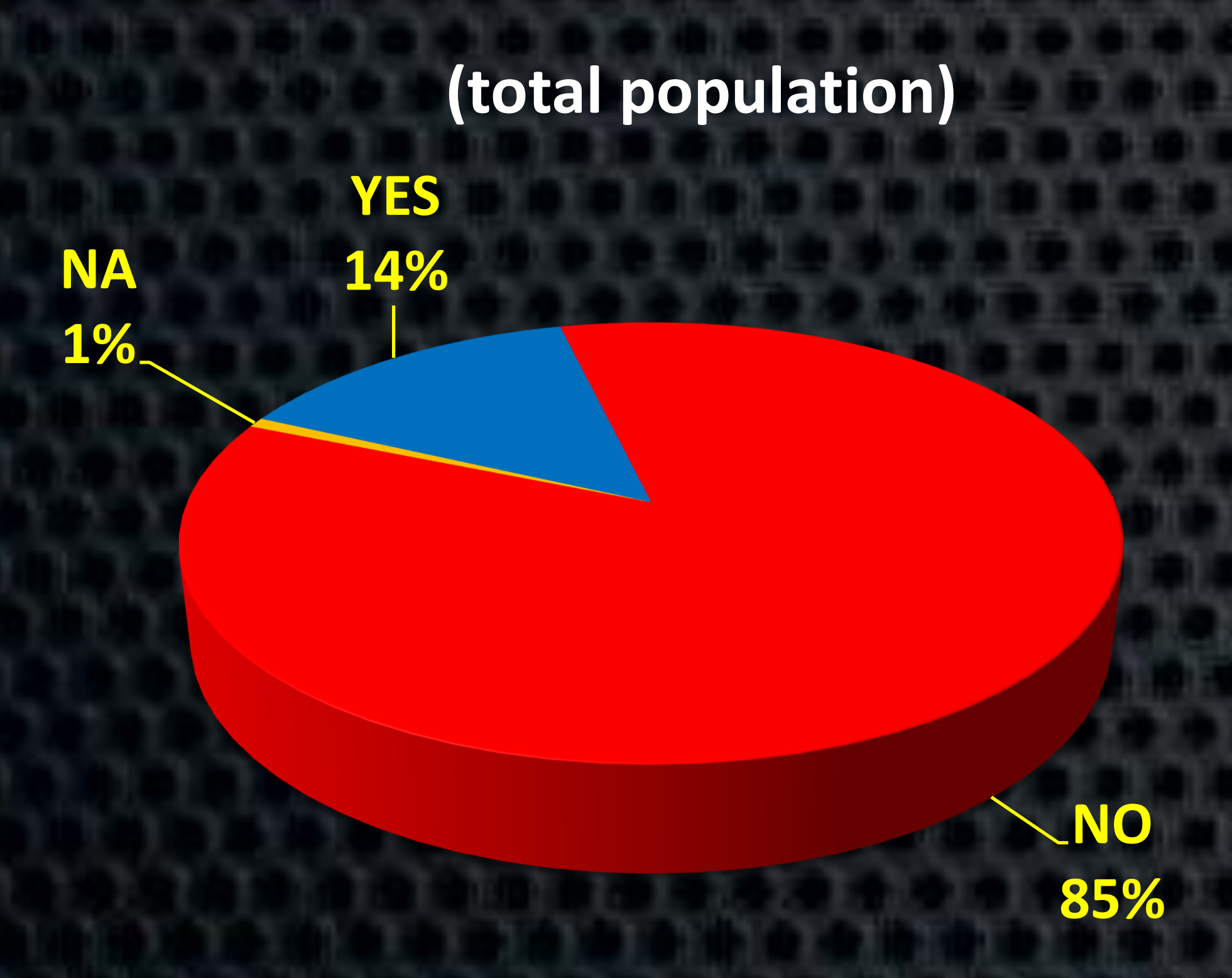

Which of the following

causes diabetes?

(total population)

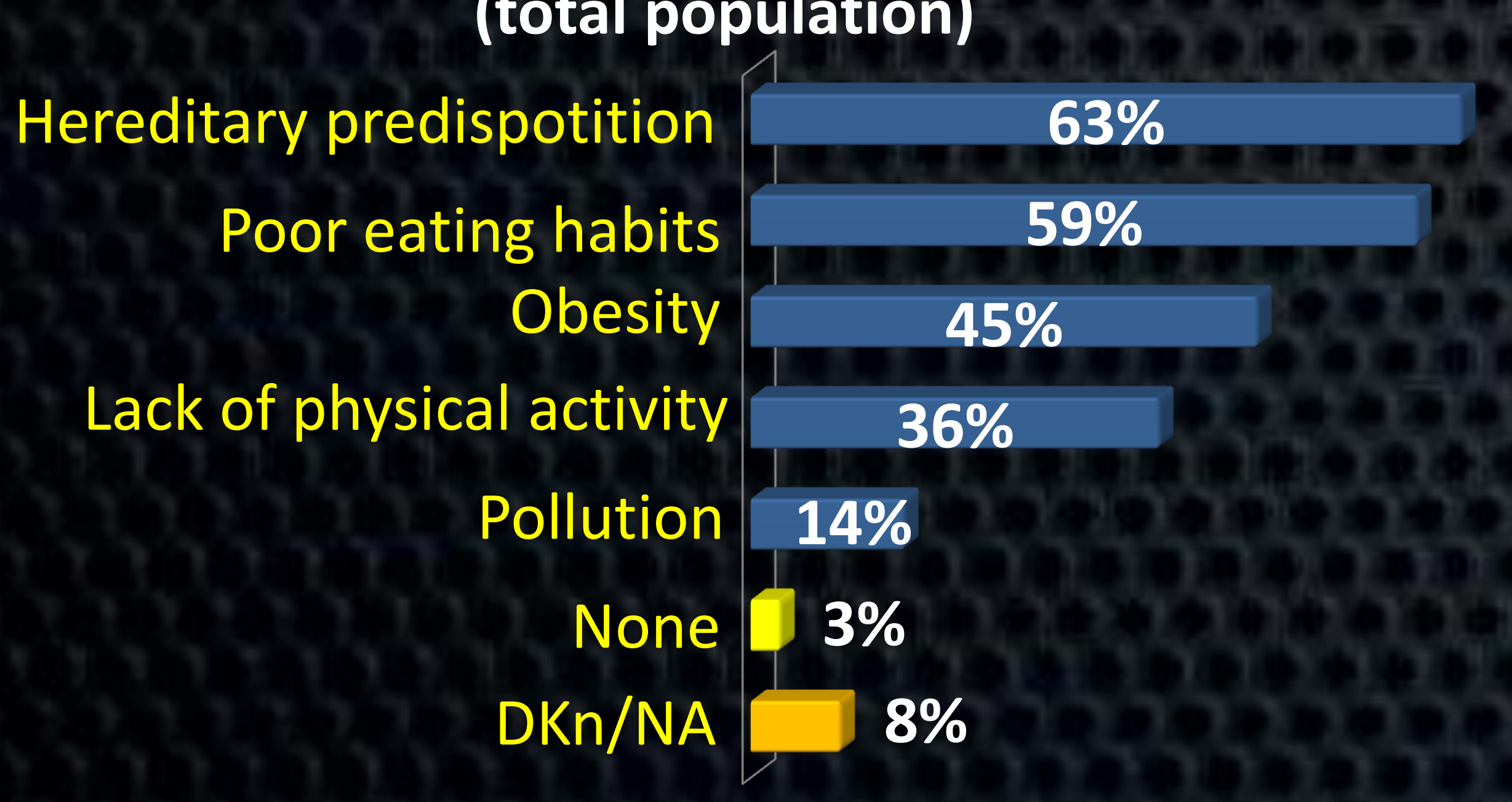

Is there a person with diabetes in your family environment? (among those without diabetes, $N=509$ )

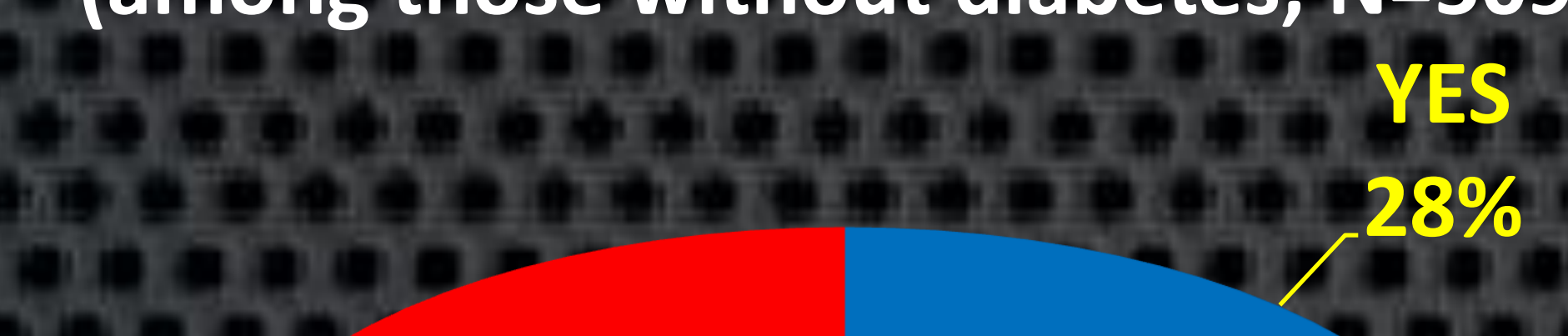

No

$72 \%$

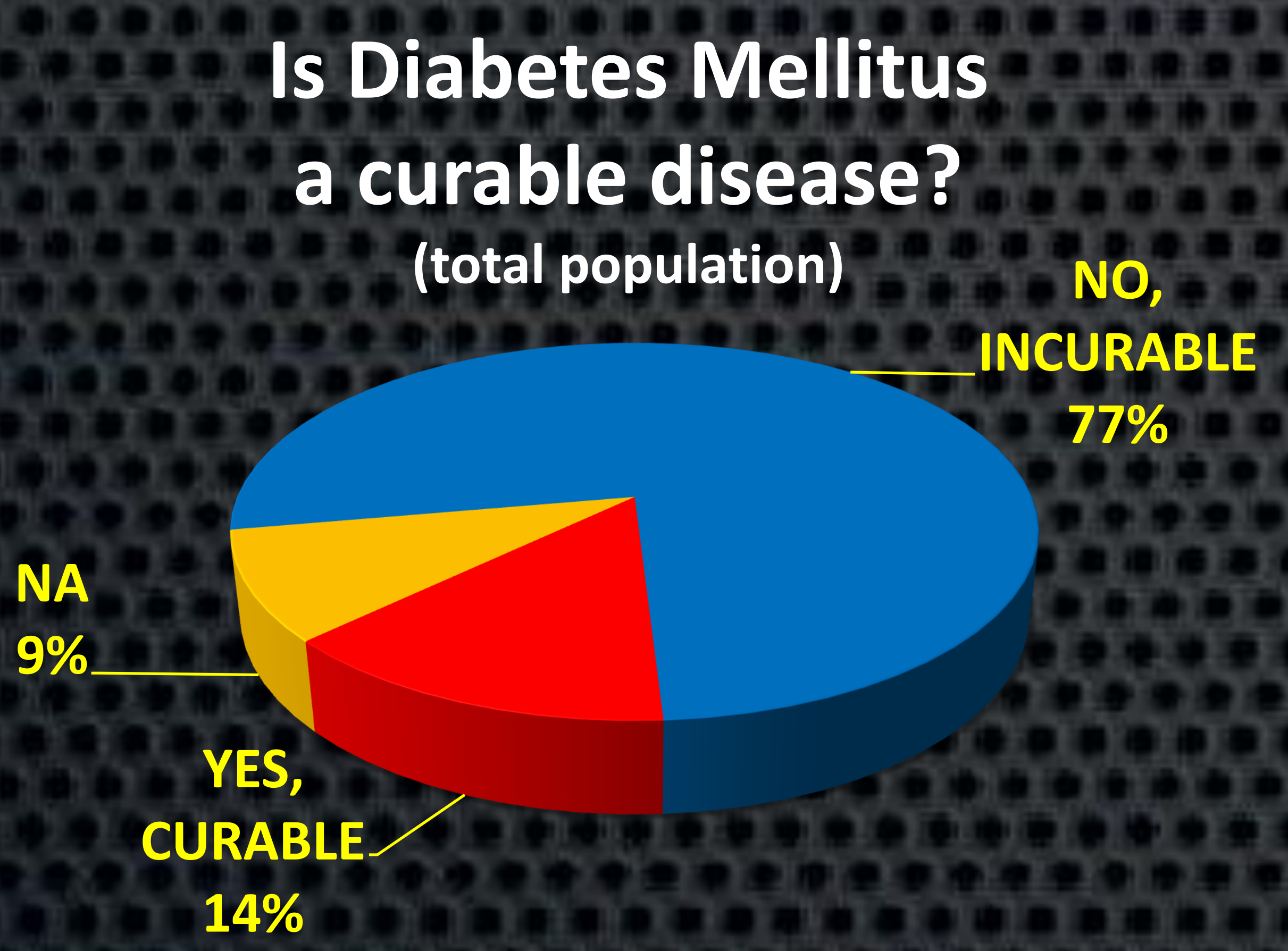

What would be your first choice of phycisian for diabetes management? (total population)

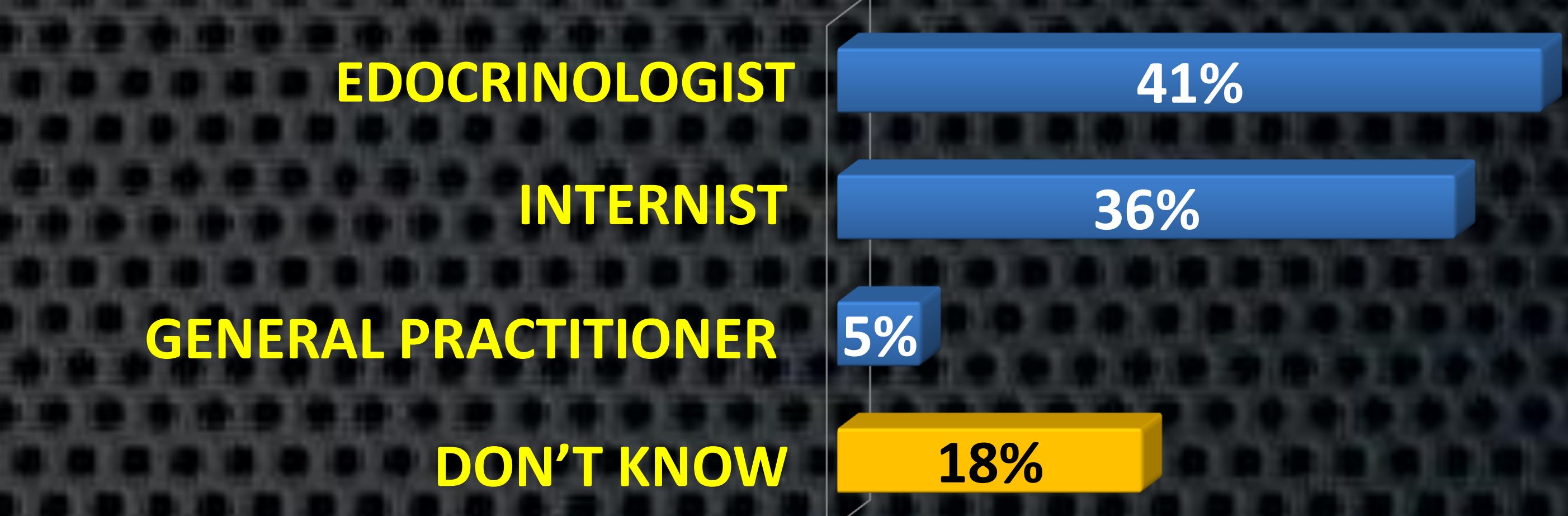

DON'T KNOW

Compliance to medication (patients with diabetes, $\mathrm{N}=\mathbf{7 8}$ )

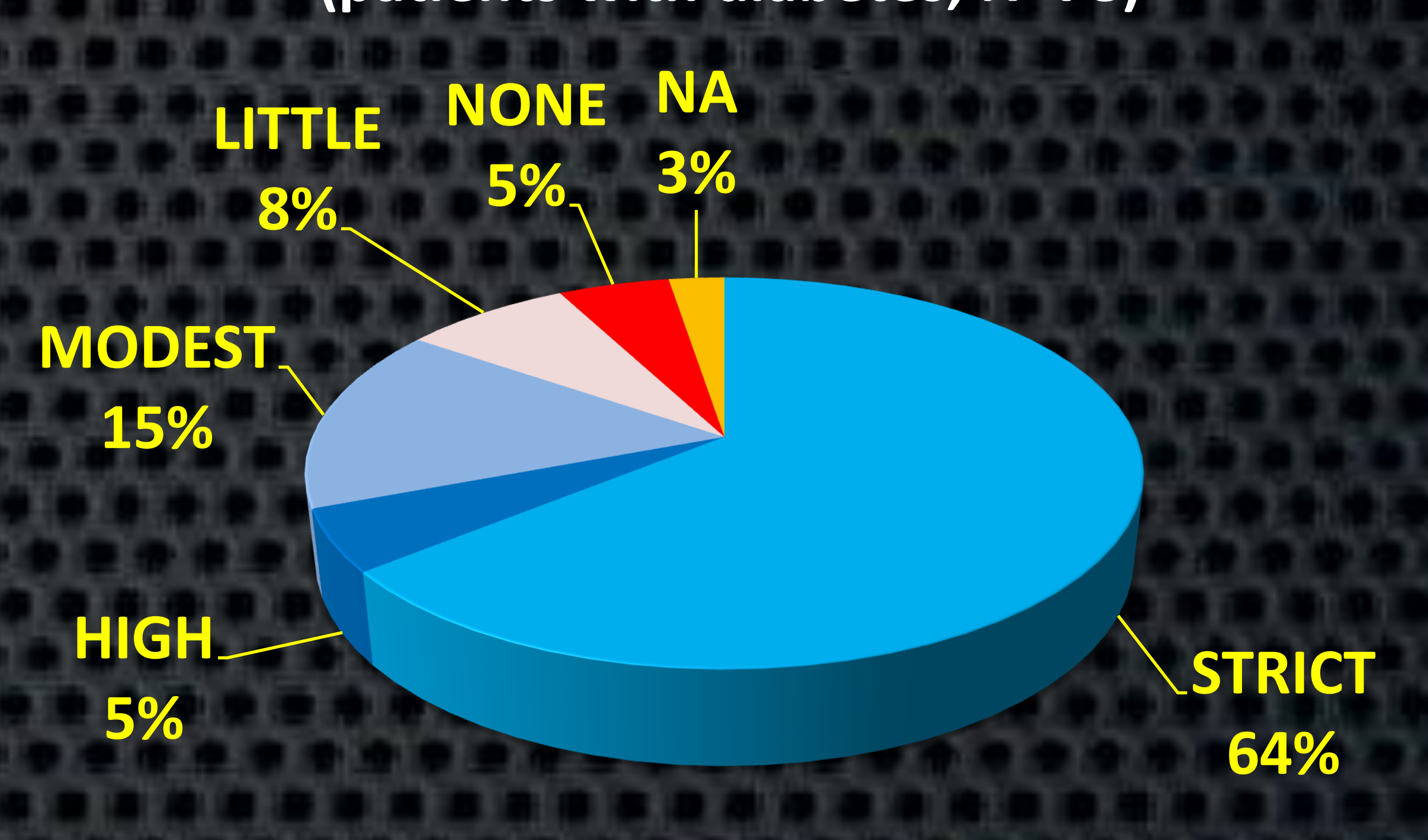

Conclusion: We believe that the level of awareness of diabetes in northern Greece is satisfactory. Communication to the general population of the main predisposing factors especially obesity and the importance of compliance to treatment in order to optimize treatment and avoid complications are needed. 